\title{
Prevalence and determinants of Anemia among pregnant women in sub-Saharan Africa: a systematic review and Meta- analysis
}

Meseret Belete Fite ${ }^{1 *+} \mathbb{D}$, Nega Assefa ${ }^{2+}$ and Bizatu Mengiste ${ }^{2+}$

\begin{abstract}
Background: Anemia is one of the world's leading cause of disability and the most serious global public health issues. This systematic review and meta-analysis was carried out very prudently in order to give up the pooled prevalence and determinants of anemia in Sub-Saharan Africa.

Methodology: To carry out this ephemeral systematic review and meta-analysis, a correlated literature review was done from various sources, PubMed Medline and Google Scholar Journals. Anemia related searching engine was used to make the study more evocative and intensive. We used modified Newcastle-Ottawa quality assessment scale for cross sectional studies to evaluate the quality of the study in relations of their inclusion. The Preferred Reporting Items for Systematic Reviews and Meta-Analyses (PRISMA) guideline was tracked to conduct this study. The pooled effect size was computed using the review manager and Compressive Meta-analysis software.

Results: Twenty-fife studies, which encompassed 15,061 pregnant women, were chosen for the analysis. From those an overall prevalence of anemia in pregnancy in SSA was 35.6\%. However, the result from meta-analysis showed that women who were infected with intestinal parasite were 3.59 times more likely to develop anemia compared to those who were not infected [OR:3.59, 95\% Cl (2.44,5.28)].The result showed that women who had no iron and folic-acid supplementation were 1.82 times more likely to develop anemia compared to those women who had iron and folic-acid supplementation $\{\mathrm{OR}: 1.82,95 \% \mathrm{Cl}(1.22,2.70]$. Women who had women were in third trimester pregnancy were 2.37 times more likely to develop anemia compared to those who were in first and second trimester [OR:2.37, 95\% Cl $(1.78,3.24)]$. Women who had low dietary diversity score were 3.59 times more likely to develop anemia compared to those who had high dietary diversity score [OR: 3.59, 95\% Cl $(2.44,5.28]$.
\end{abstract}

\footnotetext{
* Correspondence: meseretphd2014@gmail.com

${ }^{\dagger}$ Meseret Belete Fite, Nega Assefa and Bizatu Mengiste Alemu contributed equally to this work.

${ }^{1}$ Department of Public health, Institute of Health Science, Wollega University, Nekemte, Ethiopia

Full list of author information is available at the end of the article
}

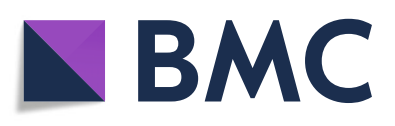

(c) The Author(s). 2021 Open Access This article is licensed under a Creative Commons Attribution 4.0 International License, which permits use, sharing, adaptation, distribution and reproduction in any medium or format, as long as you give appropriate credit to the original author(s) and the source, provide a link to the Creative Commons licence, and indicate if changes were made. The images or other third party material in this article are included in the article's Creative Commons licence, unless indicated otherwise in a credit line to the material. If material is not included in the article's Creative Commons licence and your intended use is not permitted by statutory regulation or exceeds the permitted use, you will need to obtain permission directly from the copyright holder. To view a copy of this licence, visit http://creativecommons.org/licenses/by/4.0/. The Creative Commons Public Domain Dedication waiver (http://creativecommons.org/publicdomain/zero/1.0/) applies to the data made available in this article, unless otherwise stated in a credit line to the data. 
Conclusions: Our finding from this systematic review and meta-analysis displays the high case in prevalence of anemia among pregnant women in Sub-Saharan Africa. Predictors for this includes: intestinal parasite, iron and folic-acid supplementation, third trimester pregnancy and dietary diversified intake score were statistically correlated positively with anemia in pregnancy. These need cautious evaluation of impact of prevention effort for operational policy, programs and design nutrition intrusions for refining maternal food consumption during pregnancy. Also, dietary education intrusion requires to be prearranged to satisfy the desires of pregnant women. The finding of this work will be used as an evidences for policy makers of Africa; entirely for maternal and child health care. Lastly, we suggested further investigations to be carried out in the area of the study for more rigorous and comprehensive recommendations.

Keywords: Prevalence of anemia, Intestinal parasite, iron and folic-acid supplementation, Systematic review, metaanalysis, Sub-Saharan Africa

\section{Introduction}

In all over the world, anemia is one of the public health problems and continued as a universal top cause of frailty and the uppermost serious global health issues. This is because in a pregnancy, it is tremendously major both in industrialized and unindustrialized countries. Current suggestion from World Health Organization (WHO) document showed that, about 38\% (32 million) of pregnant women are anemic in the word. Out of this, 46.3\% (9.2 Million) of them are in Africa [1]. Nevertheless, the explanations of the rate frequently display inconsistency in the world from place to place [2].. For example, there is considerable variation in the rate of anemia during pregnancy within developed countries like United States in which the rate is $18 \%$, in Australia 20\%, in Singapore $67.8 \%$ and in China $70 \%$; while the rates upsurge over trimesters [3-5]. However, the extent of the rate is becoming greater in developing countries; for instance, in Ethiopia 50.1\%, in Sudan 53\%, in Guinea $71 \%$ and in Pakistan $76.7 \%$. These are the basic rationale problems associated to anemia, which is one of the fundamental concerns of public health issues in the world in over-all and in Africa in specifically [6-8].

Finding from a number of studies carried out on this present issue displayed that anemia in pregnancy has been related to adverse pregnancy outcome and fetal growth [9]. These includes premature birth, low birth weight, abortion, delay psychomotor improvement, impairment of cognitive recital and reduce totals on intelligence (IQ) test level of the newly born baby which has an effect on the later life of the children at all [1016]. Furthermore, the impact of Iron Deficiency Anemia (IDA) in first stages of teenager and early youthful are not probably to be adapted by considerable iron administration [14]. This is because the iron dietary consumption upraises maternal mean hemoglobin concentration reads from 4.59 to $5.46 \mathrm{~g} / \mathrm{L}$. Therefore, excessive intake of dietary iron at first or successive trimester pregnancy is meaningfully associated with decrement of the threat of anemia. This results in decreases of adverse birth outcome, premature birth and LBW [6]. Equally, women in Sub-Saharan Africa (SSA) consume low dietary iron, Calcium and Folic-Acid having less than Recommended Dietary Allowances (DRA) requirements for a woman during pregnancy for the reason that they were economically not recognized $[15,16]$.

Various studies had examined multiple aspects upsetting anemia in pregnancy. The independent predictors which include maternal age, residence, literateness, antenatal care visit, inter-pregnancy interval, iron food consumption, dietary practice, micronutrient intake, dietary diversity, iron supplementation, parasite infection and gravidity were documented as factors associated with developing anemia in pregnancy [17-19]. The finding of the study suggested that women of third trimester pregnancy are more likely risky to develop anemia as compared to first and second trimester [20]. World Health Organization recommends day-to-day supplementation of $30-60 \mathrm{mg} / \mathrm{d}$ elemental iron $(+400 \mu \mathrm{g})$ and folic acid to reduce the burden of anemia as a public health problem [1]. Finding of different studies also presented that compliance to Iron and Folic-Acid Supplementation (IFAS) in Sub-Saharan Africa countries has a better position to some degree. However the problem is silent leftovers at subnormal level in which compliance percentage arrays from $10.6 \%$ in Kenya to $79 \%$ in Mozambique [21, 22].

A number of lately published studies on adherence with IFAS in pregnancy in Sub-Saharan African counties are recognized [23-47], but there is no systematic review and meta-analysis conducted on prevalence of anemia and its determinants in SSA. Furthermore, the present overall prevalence of anemia in pregnancy is not wellknown in this setup empirically. Hence, in order to sum up studies carried out in different angles of SSA countries and give overall prevalence of anemia and its determinants; this systematic review and meta-analysis was done cautiously to alleviate the problem. 


\section{Methods}

To conduct this brief systematic review and metaanalysis, a related literature of articles from PubMed, Medline and Google Scholar journal data base were collected. To increase the probability of all-inclusiveness of the findings, uniterms and Boolean operators in English were used in searching strategies. Terms used for searching were: The search terms used were; "anemia OR anemia during pregnancy OR determinants of anemia" and name of African countries include: Kenya, Sudan, South Sudan, Ethiopia, Gabon, Nigeria, Ghana, Uganda, Benin, Somalia, Eretria, Malawi, Djibouti and so on. Finally, the results of this review were reported based on the Preferred Reporting Items for Systematic Review and Meta-Analysis statement (PRISMA) guideline.

\section{Selection of the studies}

All articles related to prevalence and determinant of anemia were collected from various sources. Since the year of publication for each article were not restricted, all studies published up to February 25, 2020 were included for their eligibility in the review. Then, quantitative cross-sectional study design was used to make the study stronger and more meaningful. Nevertheless, studies published in qualitative methods were excluded due to the feature of the review and analysis selected to be used in this paper. Then to have a deep understanding of each article, all authors read the title and abstract part independently. To avoid biases, all eligible studies were screened and chosen after all individual's full reading of the abstract section of each study. Then the disagreement of the work was managed to increase the reliability and validity of the review and analysis based on pre-set inclusion criteria. The reference lists of the studies screened for the systematic review and meta-analysis were surveyed to trace citations of references.

\section{Data extraction and quality assessment}

All authors were participated in the data extraction. Data extraction template, which included author's name, year of publication, study location, sample size, odds ratio, confidence intervals and $P$-value, was prepared before the extraction of data was carried out. After the extraction of the data by each individual independently, we made a cross checked and compared the results. All authors discussed and came to consensus on little partiality observed during the work. The Modified NewcastleOttawa quality assessment scale for cross-sectional studies was used to assess the quality of the studies in terms of its inclusion. The total score for the modified Newcastle-Ottawa scale for cross-sectional studies used was nine [9] stars as a maximum for the overall scale with the minimum of zero, and a study was considered to be a high quality if 7 was achieved from 9 and medium if 5 was achieved from 9 [48].

\section{Outcome interests}

The primary outcome of this study was anemia in pregnancy period of the woman. Variables such as Age, Family size, income and parity were sought, but no sufficient information were obtained from published studies. Potential factors affecting the anemia includes: intestinal parasite, iron and folic-acid supplementation, third trimester pregnancy and Dietary Diversified Score (DDS). Thus, dietary diversity score was measured by collecting evidence on dietary consumption by means of 24-h dietary recall method. The score is classified as low $(\mathrm{DDS} \leq 3)$, medium (DDS $=4$ or 5$)$ and high $(\mathrm{DDS} \geq 5)$. Final clarification is the fourth visit for antenatal care which is defined as pregnant women who received antenatal care four or more times during the pregnancy period.

\section{Statistically analysis}

The extracted data was copied to Microsoft excel to be exported to review manager version 5.3 and the compressive meta-analysis version 2 software for careful analysis. Accordingly, statistical description related to anemia and its determinants were performed. Sensitivity analysis recognized five articles that were considered outliers, distortion and have made over estimation in the pooled effect size measurement were removed and excluded from the final meta-analysis. When the required information was not presented in the articles, the authors of the studies were contacted with their email address. Risk of bias for each study was carefully assessed. Accordingly, the trim-and-fill test was used for crude association to identify the possible effect of absences of studies from meta-analysis of the overall pooled effect size. All selected articles for this study have reported the Odd rations and helped the bias come from the converting. Regarding of lessening the information bias, alternative encouraging way was the use of original investigations that gained information from reliable exposure and outcome measures, such as laboratory tests and clinical records, rather than self-reported information.

The existence of statistical heterogeneity and the publications bias were tested by funnel plot and empirically through Egger's regression test. The degree of trustworthiness was contemplated. The heterogeneity of studies was computed using the I-squared statistic. In this process, 25\% was signified as low, 50\% moderate and $75 \%$ as high heterogeneity score. Subgroup analysis was executed by the study sub-region and study type (Community based and / or facility based). The effect of particular predictor's variables which consist of: 
intestinal parasite, iron and folic-acid supplementation, third trimester pregnancy and dietary diversified Score and the result of the meta-analysis revealed forest pilot and Odd Ratio (OR) with 95\% of CI.

\section{Result}

\section{Studies selection}

Based on the objectives set for this work, we identified different studies related to the prevalence and determinants of anemia for the inclusion in meta-analysis before directly move to the other detail part of this paper. Accordingly, we found 1256 completed studies published on international journals. From these, 1313 of them were excluded for they were not satisfying the criterion of inclusion set in this study. However, 53 articles were chosen from those studies for their eligibility. Out of these 28 studies were rejected due to their poor statistical reports and defect of data observed in each of them. Finally, only 25 studies were added in this analysis for their neatness and clear justification. (Fig. 1).

\section{Characteristics of included studies}

Twenty-three cross-sectional studies from different countries of Africa were included in the meta-analysis (23-47) (Table 1). Out of those two of them (8\%) were from Kenya, four (16\%) from Ghana, two (8\%) from Nigeria, two (8\%) from Uganda, one (4\%) from Benin, and fourteen (56\%) from Ethiopia. Among those the highest sample size was observed in studies conducted in Benin [34] which was equal 3519 and the lowest was found in Kenya, 258 [47]. The mean age of the respondents was 27 years. Out of twenty-five studies incorporated in this review and analysis, though twenty-one studies (24-31, 39, 41-47) were conducted on facility based, four of them $(\mathbf{1 4}, \mathbf{2 3}, \mathbf{2 9}, \mathbf{3 1})$ were done on community-based work.

\section{Prevalence of Anemia}

From the analysis made, the lowest prevalence of anemia (7.9\%) was observed in Ethiopia [25], but the highest compliance, which is equal to $(76 \%)$ was observed in

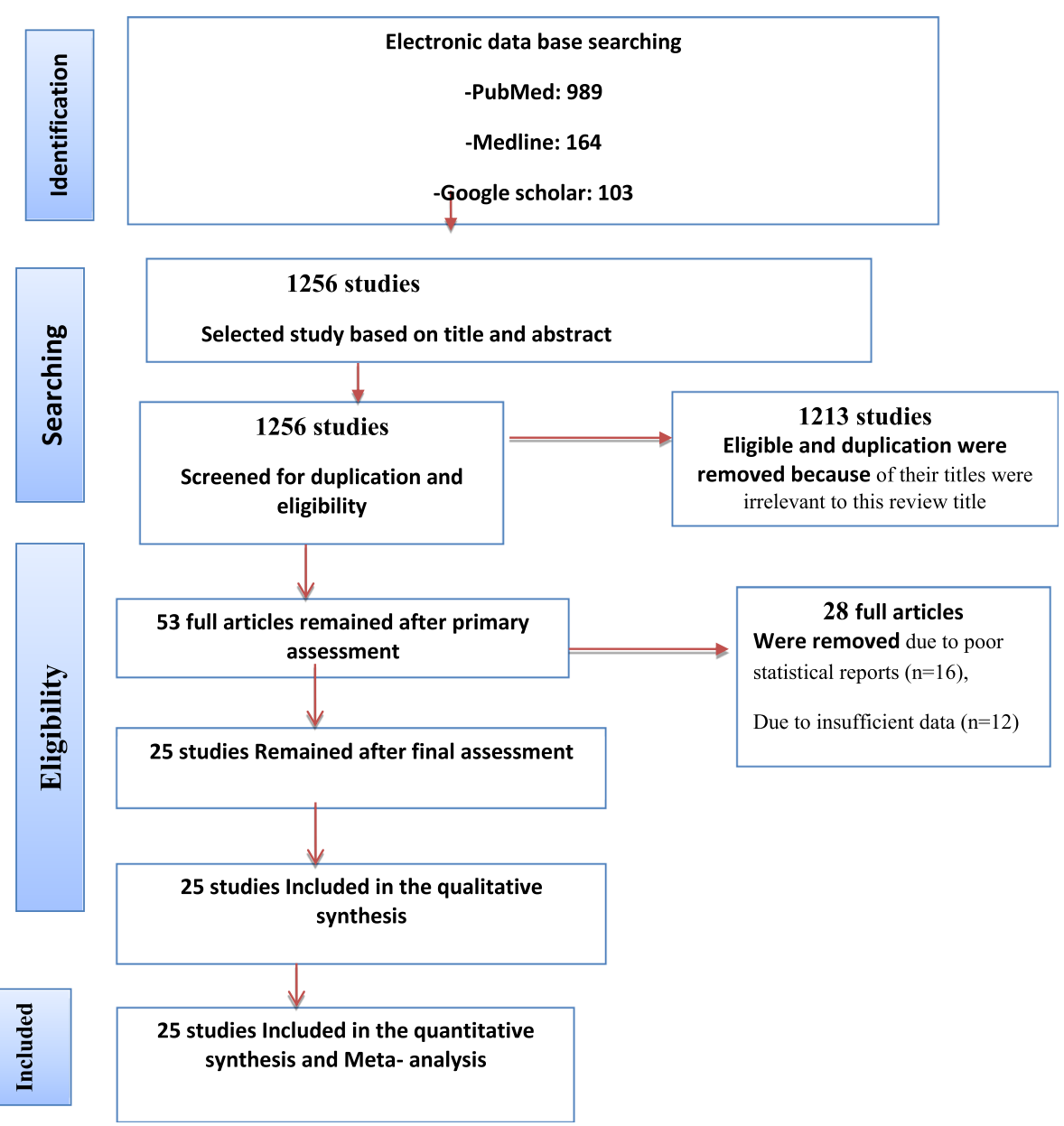

Fig. 1 Flow diagram of the studies included in the Meta-analysis 
Table 1 Characteristics of studies included in systematic review of prevalence compliance with IFAS among pregnant women in Sub-Saharan Africa

\begin{tabular}{|c|c|c|c|c|c|}
\hline Author & Region & Study design & Study type & Sample Size & Prevalence in $\%$ \\
\hline Addis Alene and A. Mohamed Dohe & Ethiopia & Community based & Community based & 577 & 56.8 \\
\hline Adediran et al & Nigeria & Facility based & Facility based & 170 & 46.6 \\
\hline Angesom Gebreweld and Aster & Ethiopia & Facility based & Facility based & 228 & 11.6 \\
\hline Berhe et al & Ethiopia & Facility based & Facility based & 304 & 7.9 \\
\hline Bolka and Gebremedhin & Ethiopia & Facility based & Facility based & 349 & 31.5 \\
\hline Derso et al & Ethiopia & Facility based & Facility based & 348 & 30.5 \\
\hline Freda Dzifa Intiful et al.,2016 & Ghana & Facility based & Facility based & 265 & 76 \\
\hline Getachew et al & Ethiopia & community based & community based & 393 & 53.9 \\
\hline Grum et al & Ethiopia & Facility based & Facility based & 634 & 16.88 \\
\hline Judith K. Anchang-Kimbi et al.,2016 & Ghana & Facility based & Facility based & 320 & 66.6 \\
\hline Judith Koryo Stephens et al & Ghana & Facility based & Facility based & 316 & 41.5 \\
\hline Kefiyalew et al & Ethiopia & Facility based & Facility based & 258 & 27.9 \\
\hline Lealem G. et al & Ethiopia & Facility based & Facility based & 363 & 39.94 \\
\hline Lebso et al & Ethiopia & community based & community based & 507 & 23.2 \\
\hline Mengist et al & Ethiopia & & & 372 & 17.5 \\
\hline Nega et al. & Ethiopia & community based & community based & 341 & 34.6 \\
\hline Niguse $O$ et al & Ethiopia & Facility based & Facility based & 374 & 36.6 \\
\hline Nonterah EA, Adomolga E, Yidana A et al & Ghana & Facility based & Facility based & 506 & 42.7 \\
\hline O. T. Okube et al & Kenya & Facility based & Facility based & 258 & 57 \\
\hline Obai et al & Uganda & Facility based & Facility based & 743 & 22.1 \\
\hline Ononge et al & Uganda & Facility based & Facility based & 2436 & 32.5 \\
\hline Ouédraogo et al & Benin & Facility based & Facility based & 3519 & 68.2 \\
\hline Shitie et al & Ethiopia & Facility based & Facility based & 284 & 2.8 \\
\hline Uneke C. J et al. & Nigeria & Facility based & Facility based & 815 & 76.9 \\
\hline Wanjiru C. & Kenya & Facility based & Facility based & 381 & 36.2 \\
\hline
\end{tabular}

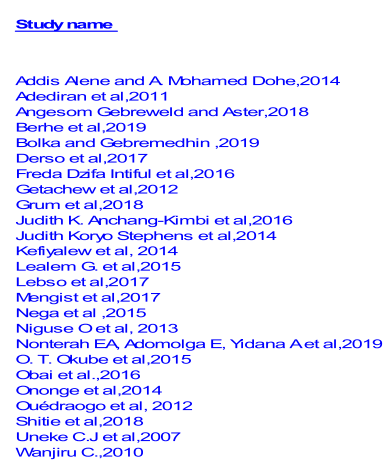

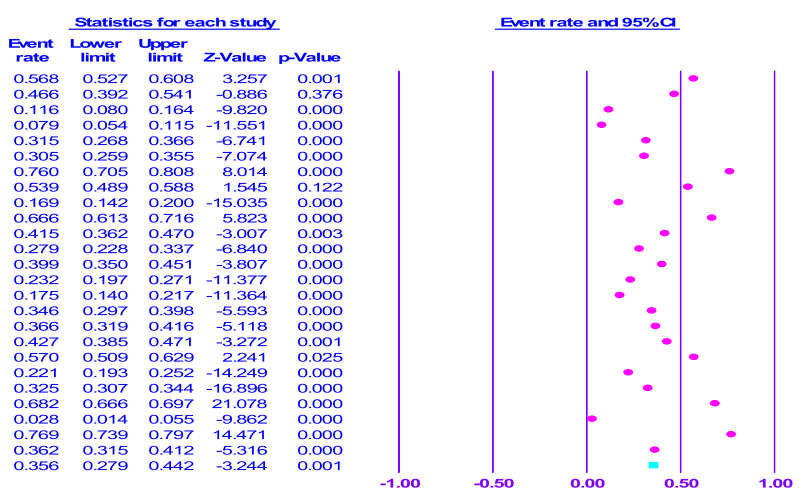

Fig. 2 Forest plot displaying the pooled prevalence of anemia among pregnant women in Sub-Saharan Africa 
study conducted in Ghana [31]. On the other hand, the pooled prevalence of anemia amongst pregnant women in SSA was $35.6 \%(95 \% \mathrm{CI}=0.279-0.442)$ (Fig. 2).

\section{Subgroup analysis}

A subgroup analysis was done by classifying studies based on corresponding sub-regional location in SubSaharan Africa in order to compute and relate the prevalence of anemia focusing on athwart various participants' characteristics. Based on this, the lowest prevalence of anemia in pregnancy was documented in Eastern Africa, (33.9\%) (CI: 0.252, 0.438) and the highest prevalence of anemia was recognized in Western Sub-Saharan, (39.3(CI: 0.231, 0.582). However, a greater prevalence of compliance, which is equal to $(41.4 \% \%)$ was detected in studies conducted at facility level than community level (CI: 0.262, 0.584) (Table 2).

\section{Association of intestinal parasite with anemia}

Out of twenty-five chosen studies conducted on the area of the key concern and included in the meta-analysis, in nine of them (31-39), it was documented that infection of intestinal parasite was associated with anemia in pregnancy. Moreover, the result from meta-analysis also revealed that women who were infected with intestinal parasite were 3.59 times more likely to develop anemia compared to those who were not infected [OR:3.59, 95\% CI $(2.44,5.28)]$. Thus, the heterogeneity test revealed $\mathrm{I}^{2}=68 \%$ and the statistical evidence of this is $P \leq 0.00001)$. From this we can understand that the random-effect analysis was the secondhand one. Thus, the Bag's and Egger's test for publication bias indicated that there is no statistical evidence of Publication bias. That is their $p$-values are equal to 0.117 and 0.05 respectively (Fig. 3).

\section{Association of Iron Folate Supplementation with anemia} The association of lack of iron folic-acid supplementation and risk of developing anemia during pregnancy was stated in seven chosen studies [23, 26, 29, 38, 39, 46, 47]. The result of meta-analysis from exhibited that women who had no iron folic-acid supplementation were 1.82 times more likely to develop anemia compared to those women who had iron folic-acid supplementation \{OR:1.82, 95\% CI (1.22,2.70] (Fig.4). The heterogeneity test indicated $(\mathrm{I} 2=68 \%)$ and statistical evidence of this heterogeneity was $P \leq 0.003$ ). Hence, the randomeffect analysis was carried out. The Bag's and Egger's test for publication bias indicated that there is no statistical evidence of publication bias, which is equal to 0.76 and 0.85 (Supplementary 1).

\section{Association stages of pregnancy (trimester) with anemia}

The important analysis was focus on the association of pregnancy stage and risk of developing anemia. This was stated in eight studies [23, 25, 26, 28, 37, 41, 42, 47]. The result of meta-analysis showed that women who were in third trimester pregnancy were 2.09 times more likely to develop anemia compared to those who were in first and second trimesters [OR:2.09, 95\% CI (1.60, $2.74)\}$. The heterogeneity test indicated that $\left(\mathrm{I}^{2}\right.$ is equal to $58 \%$ and the statistical evidence of this is $P \leq 0.0001$ ). Hence, the random-effect analysis was carried out. The Bag's and Egger's test for publication bias indicated that there is no statistical evidence of publication bias, which is equal to 0.17 and 0.12 respectively (Fig.5).

\section{Association of Dietary diversity score with anemia}

The association between Dietary diversity score and risk of developing noncompliance to IFAS during pregnancy was stated in eight studies [25, 28, 30, 31, 36, 37, 40, 43]. The result from meta-analysis from those revealed that women who had low Dietary Diversified intake Score (DDS) were 3.59 times more likely to develop anemia compared to those who had high DSS [OR: $3.59,95 \% \mathrm{CI}$ $(2.44,5.28]$. The heterogeneity test indicated $\mathrm{I}^{2}=68 \%$ and statistical evidence of this was $\leq 0.00001$ ). Therefore, the random-effect analysis was secondhand performed. The Bag's and Egger's test for publication bias indicated that there is no statistical evidence of publication bias, which is equal to 0.89 and 0.25 consecutively (Fig.6).

Table 2 Subgroup analysis of prevalence of aneemia among pregnant women in Sub-Saharan Africa

\begin{tabular}{|c|c|c|c|c|c|c|}
\hline Subgroup & No. of included studies & Prevalence(95\%Cl) & Heterogeneity Statistics & Tau Squared & $P$ value & $\mathrm{I}^{2}$ \\
\hline \multicolumn{7}{|l|}{ By Sub- region } \\
\hline Eastern Africa & 17 & $33.9(0.252,0.438)$ & 1273.69 & 0757 & $<0.000$ & 98.85 \\
\hline Western Africa & 8 & $39.3(0.231,0582)$ & 877.78 & 0.856 & $<0.000$ & 98.35 \\
\hline \multicolumn{7}{|l|}{ Overall } \\
\hline \multicolumn{7}{|l|}{ By study type } \\
\hline Facility based & 21 & $41.4(0.262,0.584)$ & 2150.608 & 0.903 & $<0.000$ & 99.07 \\
\hline Community based & 4 & $34.5(345,0.258)$ & 97.35 & 0.389 & $<0.000$ & 96.91 \\
\hline
\end{tabular}




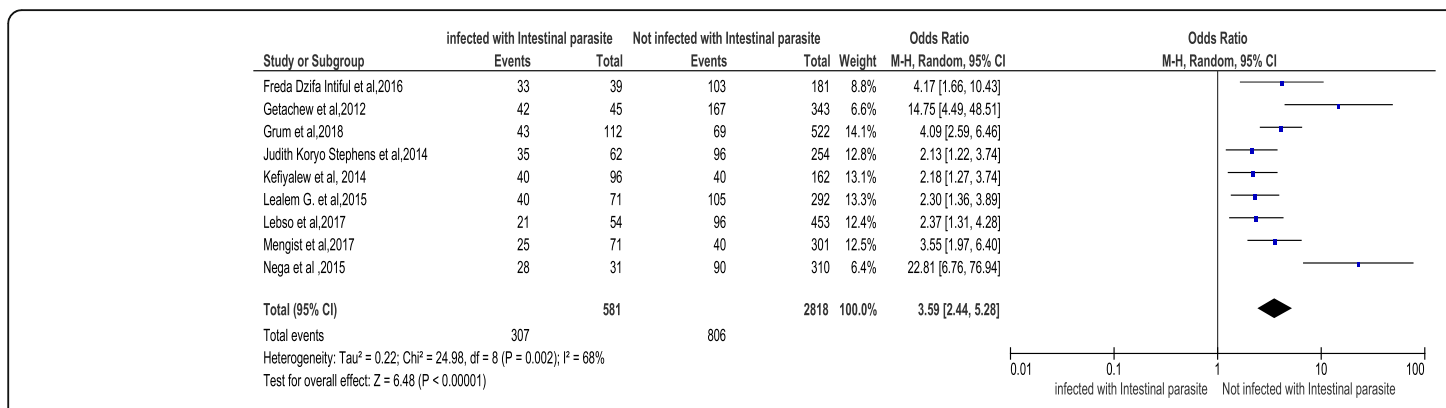

Fig. 3 Forest plot displaying Association of intestinal parasite with anemia among pregnant women in Sub-Saharan Africa

\section{Discussion}

Anemia is one of the globally top causes of frailty, the highest universal problem and identified public health matters in Sub-Saharan Africa [1]. Evidences from the meta- analysis stated above suggested that almost 38\% (32 million) women were victims of anemia in their course of pregnancy in the word.

The systematic review and meta-analysis presented in this paper showed the magnitude of anemia in subSaharan-Africa and its determinants. Accordingly, the key finding of analysis exhibited the anemia in pregnancy prevalence in Sub-Saharan Africa in which the pooled prevalence was equal to $35.6 \%$; however, the heterogeneity test indicates its statistical evidence which was elucidated by difference in geographic location, for instance, Eastern Africa and western Africa types of study which was focused on community and facilitybased type. In relation to this analysis, a study conducted in Iran stated that the prevalence of compliance with IFAS among pregnant women was $71.6 \%$ [47]. This systematic review and meta-analysis showed that; the magnitude of anemia among pregnant women in subSaharan-Africa and is determinants. Thus, the main finding of meta-analysis shown, anemia in pregnancy is rampant in Sub-Saharan Africa; in which the overall pooled prevalence of $36.6 \%$ was computed. Therefore, according WHO classification; anemia is moderate public health problem in Sub-Saharan Africa. Our finding differs substantially from studies conducted in developed countries; as the prevalence of anemia in Europe ranged $10-32 \%$ [17]. Compared to developed counties anemia is more prevalent in sub-Saharan Africa. This disagreement might be low socio-economic status of the region and poor dietary practice due to poor nutritional education intervention and irregular nutritional counselling in pregnancy.

It is evident that parasite infection reduces maternal hemoglobin level and contributes for higher incidence of anemia. The recent pooled meta-analysis showed that, women who were infected with intestinal parasite in their course of pregnancy were 3.59 times more likely risky to develop anemia compared to those with no history of parasite infections. Thus, this finding is comparably consistent with an investigation conducted in UK which documented that, parasite infection was significantly associated with anemia in pregnancy and one quarter of anemic mothers were infected with one or more intestinal parasite in their course of pregnancy [49]. Association of IFA supplementation was seen in this review. Women who were not supplemented with IFA in pregnancy were 1.82 times more likely risky to develop of anemia compared to their counterparts. An overflowing of studies had explored the effect of IFA supplementation on anemia. Iron use and high intake of iron was examined for the increased maternal haemoglobin and reduced risky of Iron deficiency at first and second trimester. Therefore, the finding of this study is comparably in line with different studies related to

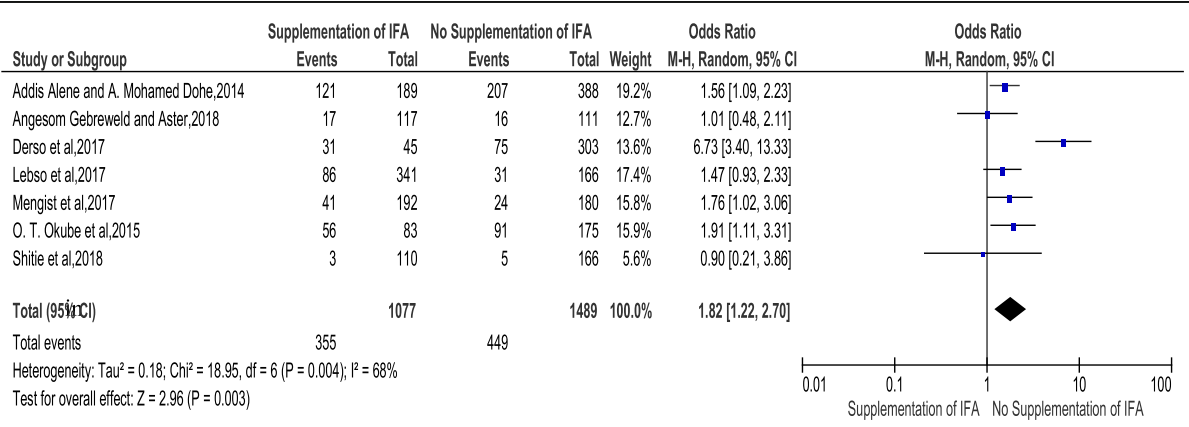

Fig. 4 Forest plot displaying Association of Iron folate supplementation with anemia among pregnant women in Sub-Saharan Africa 


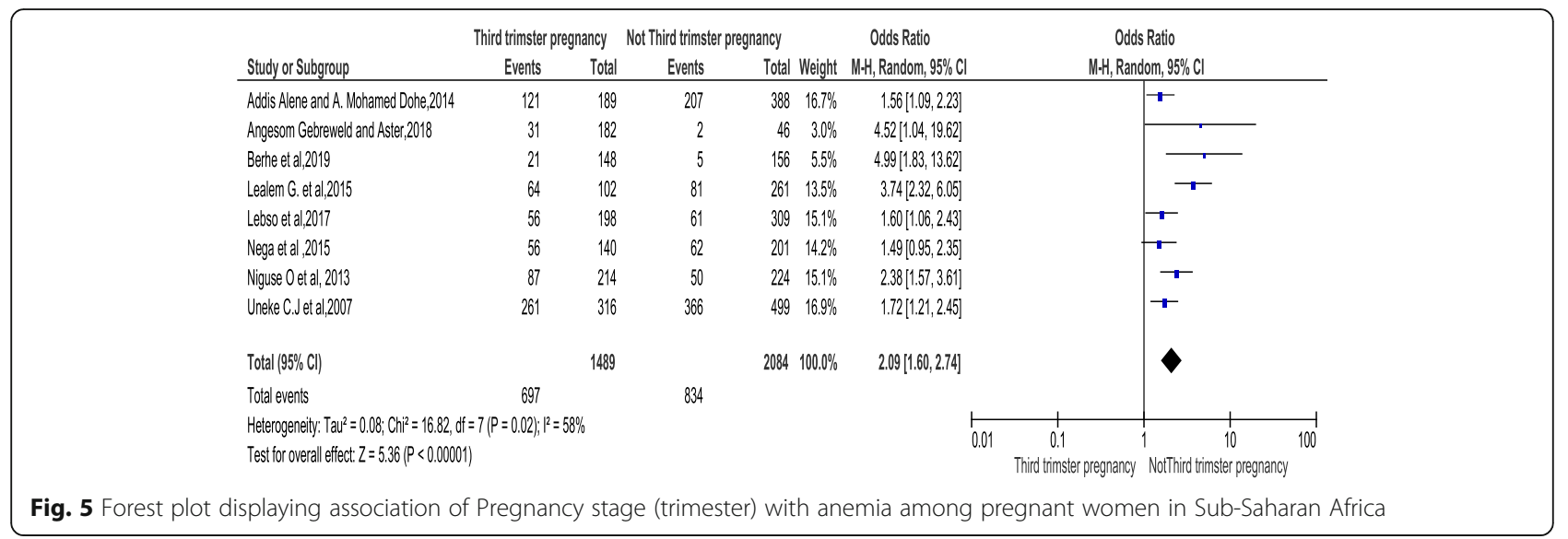

anemia which suggested that; women who are taking iron supplements have higher iron status and lower prevalence's of anemia, which are dependent on the dose of iron and compliance $[6,17,50,51]$.

Anemia is a major cause of perinatal mortally and low birth in both developed and developing countries. Studies suggested that, $40 \%$ of all perinatal deaths are linked to anemia; whereas, worthy pregnancy outcomes occur $30-45 \%$ less often in anemic mothers, and their infants have less than one-half of normal Iron reserves [52]. Low birth weight contributes $60-80 \%$ of all neonatal deaths [53]. For every $10 \mathrm{mg}$ increase in iron dose; birth weight increases by $15.1 \mathrm{~g}$ and risk of low birth weight decrease by $3 \%$ [6]. Therefore, supplementation of iron and folic acid is very important interventions for reduction of perianal mortality due to anemia. Our finding showed that, women of third trimester pregnancy were 2.37 times more likely risky to develop anemia compared to first and second trimester. In agreement to our finding, study conducted in china showed that the prevalence of anemia among pregnant women was higher during third trimester (21.8\%) than second trimester pregnancy (14.3\%) [54, 55]. Nevertheless, a prospective pregnancy cohort study carried out in African-American confirmed that, pregnant women with depleted iron stores during the 2nd trimester were 12 times more likely to be classified with iron deficiency anemia compared to their 3rd trimester (56). Thus, this variation could be due to the parasite infection and inadequate dietary practice of pregnant women which is very common in a region. Delayed for intervention and IFAS might be another reason, since most of women in developing countries lately registered in anta natal care (almost second trimester).

The current review revealed that, women with low dietary diversified intake score were 3.58 times more likely risky to develop anemia during their pregnancy. Study investigated in Canada reported that, significant relationship was found between dietary diversity score and Haemoglobin level and iron intake among pregnant women and poor dietary habit and other lifestyle

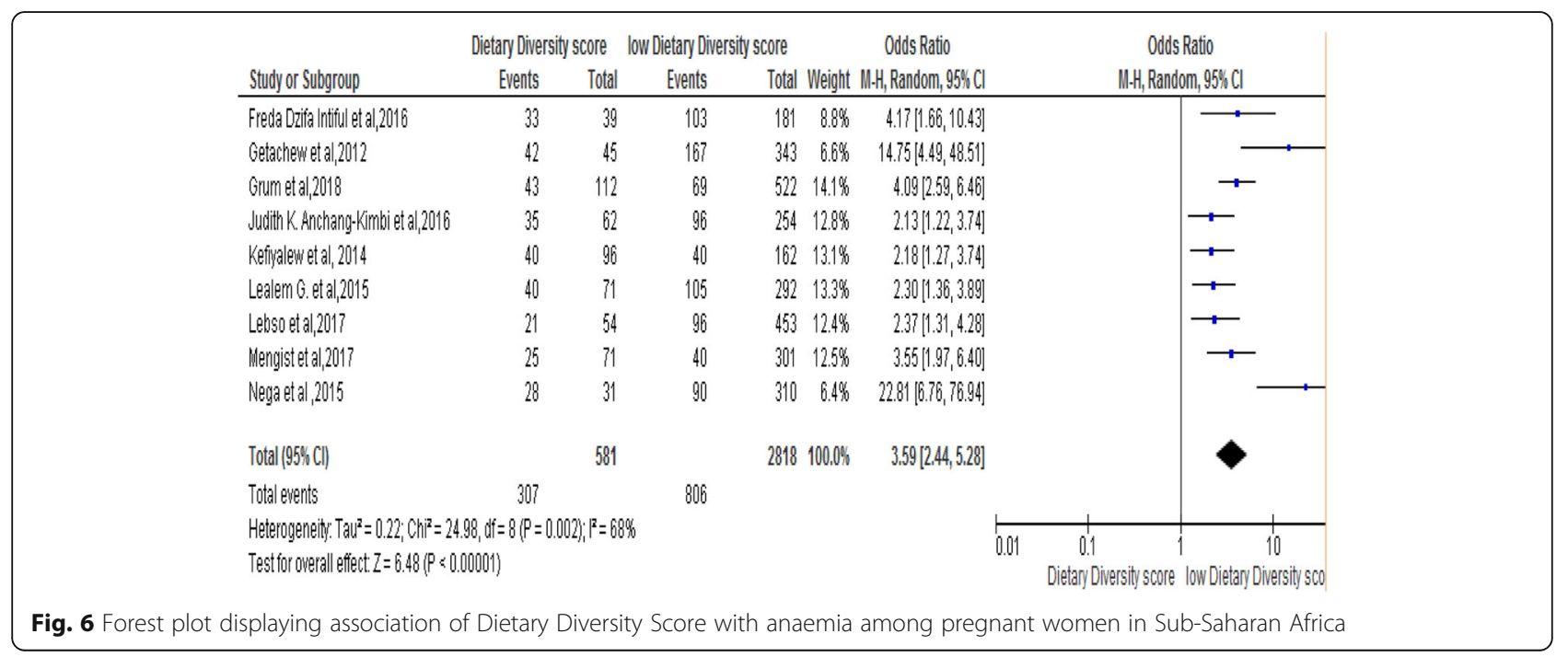


behaviour was positively associated with anemia in pregnant women [16].

However, the study lacks representativeness since there was no data found from some Sub-Saharan African counties. Also, there were no adequate studies incorporated in the analysis. Thus, this shortcoming could trouble the over-all prevalence of anemia among pregnant women in Sub-Saharan Africa.

\section{Strength and limitation}

In this review, an extensive exploration method and more than one reviewer had taken part in all courses of review process. To do so, PRISMA guideline was carefully tracked throughout the review procedure. However, the analysis has its own defects because of a number of factors. Limitations include timing, duration and compliance of iron and folic acid supplementation which could have an effect on maternal anemia were not investigated in this study. Some Factors such as income, age and parity were not intensely examined. Other limitations of the current study are lacks of representativeness due to lack of similar studies from some of Sub-Saharan African counties and some studies have been omitted because of their poor statistical reports, their small sample size and their inadequate data. Researchers have contacted authors of the published studies but some of them did not make available some pertinent information to be considered for this review. Since we considered only the crosssectional studies in the analysis, the outcome variable may possibly be affected by confounding variable and might affect the overall prevalence of anemia in pregnancy in Sub-Saharan Africa.

\section{Conclusion: implication for practice and future research}

Our finding from this systematic review and metaanalysis displays the high case in prevalence of anemia among pregnant women in Sub-Saharan Africa. Predictors for this includes: intestinal parasite, iron and folicacid supplementation, third trimester pregnancy and dietary diversity were statistically correlated positively with anemia in pregnancy. These need cautious evaluation of impact of prevention effort for operational policy, programs and design nutrition intrusions for refining maternal food consumption during pregnancy. Dietary education intrusion requires to be prearranged to satisfy the dietary desires of pregnant women.

Health care workers should have to offer dietary guidance to improve antenatal care service regularly. For instance, the recommendation from WHO reference presented that good supplementation needs making assurance that a pregnant woman is well prescribed for IFAS (lowest 90 tablets) and early registering her prior to 12 weeks of gestation period. The administration has to assign dietitian at all level of health system. Training should be programmed for health care workers who are at frontline antenatal care service at each level of health system in order to improve compliance of IFA supplementation at work place.

We hope that the finding of this work might be crucial as a bridging stone for policy makers of Africa; entirely for maternal and child health care. We suggested that further investigations to be carried out in the area of the study for more rigorous and comprehensive recommendations.

\section{Abbreviations \\ ANC: Antenatal care; WHO: World Health Organization; IFAS: Iron and Folic- Acid Supplementation}

\section{Supplementary Information}

The online version contains supplementary material available at https://doi. org/10.1186/s13690-021-00711-3.

Additional file 1. Supplementary file 1: Funnel plot displaying Publication Bias of Association of Iron folate supplementation with anemia among pregnant women in Sub-Saharan Africa. Description of figure: This figure presents, Bag's and Egger's test for publication bias showed no statistical evidence of publication bias.

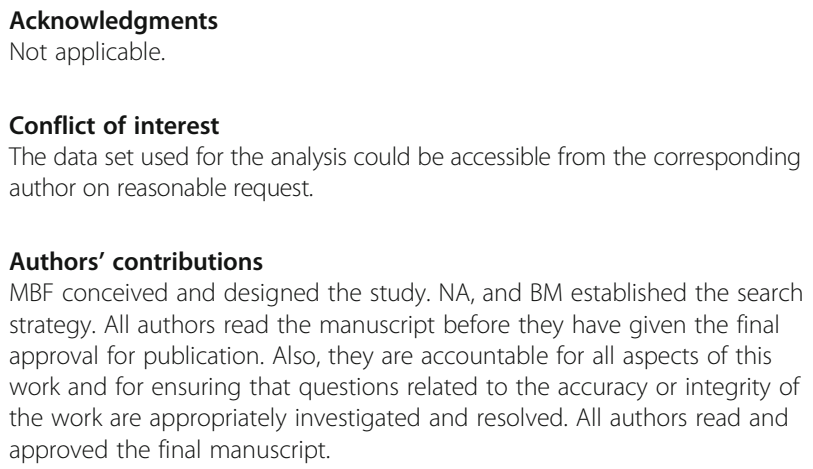

Funding

There is no received grant from any fund agency.

Availability of data and materials

All data generated or analyzed during this study are included in this published article.

\section{Declarations}

Ethics approval and consent to participate

Not applicable because no primary data were collected.

Consent for publication

Not applicable.

\section{Competing interests}

The authors declared that they have no competing interest.

\section{Author details}

${ }^{1}$ Department of Public health, Institute of Health Science, Wollega University, Nekemte, Ethiopia. ${ }^{2}$ Department of Public health, School of public health,

College of Health and Medical Sciences, Haramaya University, Harar, Ethiopia. 
Received: 24 September 2020 Accepted: 17 October 2021 Published online: 03 December 2021

\section{References}

1. WHO. Global prevalence of anemia in 2011. Geneva: Health organization; 2011.

2. WHO; de Benoist, B, McLean, E, Egli, I, Cogswell, M, editors. Worldwide prevalence of anaemia 1993-2005. WHO global database on anaemia. Geneva: World Health Organization (WHO); 2008.

3. Fakher DM, et al. Maternal serum ferritin in relation to birth weight (2015). AAMJ. 2015;13:174-9.

4. Khambalia AZ, Collins CE, Roberts CL, Morris JM, Powell KL, Tasevski V, et al. High maternal serum ferritin in early pregnancy and risk of spontaneous preterm birth (2015). Br J Nutr. 2015;114(3):455-61. https://doi.org/10.1017/ S0007114515001932.

5. Shen P, Gong B, Xu FY, Luo Y. Four trace elements in pregnant women and their relationships with adverse pregnancy outcomes. Eur Rev Med Pharmacol Sci. 2015;19(24):4690-7.

6. Haider $\mathrm{O}$, et al. Anaemia, prenatal iron use, and risk of adverse pregnancy outcomes: systematic review and meta-analysis. Bmj. 2013;346(jun21 3): f3443. https://doi.org/10.1136/bmj.f3443.

7. Fowkes FJ, et al. Iron deficiency during pregnancy is associated with a reduced risk of adverse birth outcomes in a malaria-endemic area in a longitudinal cohort study. BMC Med. 2018;16(1):156.

8. Adam I, et al. Prevalence, types and determinants of anemia among pregnant women in Sudan: a systematic review and meta-analysis. BMC Hematol. 2018:18:31.

9. Rodriguez-Bernal C, et al. Maternal nutrition and fetal growth: the role of iron status and intake during pregnancy. Nutr Diet Suppl. 2012;4:25.

10. Gogoi M. Ranjan Kumar Prusty. Guwahati: Maternal Anaemia, Pregnancy Complications and Birth Outcome; 2013.

11. Ajepe AA, Okunade KS, Sekumade Al, Daramola ES, Beke MO, ljasan O, et al. Prevalence and foetomaternal effects of iron deficiency anaemia among pregnant women in Lagos, Nigeria. PLoS One. 2020;15(1):e0227965. https:// doi.org/10.1371/journal.pone.0227965.

12. Basanti N, Romita Devi N, Devi NJ, Devi NS, Devi SDTCMTN, Meitei NM. Prevalence and determinants of severe anaemia among antenatal women attending in a tertiary care hospital in manipur. Paripex-Indian J Res. 2020; 9(1):1-4. https://doi.org/10.36106/paripex/9511147.

13. Walter T, Kovalsys J, Stekel A. Effect of mild iron deficiency on infant mental development scores. J Pediatr. 1983;102(4):519-22. https://doi.org/10.1016/ S0022-3476(83)80177-2.

14. Lozoff B, Jimenez $E$, Wolf AW. Long term developmental outcome of infants with iron deficiency. N Engl J Med. 1991;325(10):687-95. https://doi.org/10.1 056/NEJM199109053251004.

15. Tailor V, Sengupta S. Impact of Dietary Pattern on Nutritional Status of Pregnant Women in Low and High Strata between the Age Group of 30-39 Years in Mumbai. J Adv Med Medical Res. 2018:1-9. https://doi.org/10.9734/ jammr/2019/v29i830114.

16. Forbes $L E$, et al. Dietary change during pregnancy and women's reasons for change. Nutrients. 2018;10(8):1032. https://doi.org/10.3390/nu10081032.

17. Milman $\overline{\mathrm{N} \text {, Taylor }} \mathrm{CL}$, et al. Iron status in pregnant women and women of reproductive age in Europe. Am J Clin Nutr. 2017;106(Suppl 6):1655S.

18. Oaks BM, et al. Prenatal Iron deficiency and replete Iron status are associated with adverse birth outcomes, but associations differ in Ghana and Malawi. J Nutr. 2019;149(3):513-21.

19. Shen P, Gong B, Xu FY, Luo Y. Four trace elements in pregnant women and their relationships with adverse pregnancy outcomes. Eur Rev Med Pharmacol Sci. 2015;19(24):4690-7.

20. $\overline{\text { Agrawal S, et }}$ al. Adequately diversified dietary intake and iron and folic acid supplementation during pregnancy is associated with reduced occurrence of symptoms suggestive of pre-eclampsia or eclampsia in Indian women. PLoS One. 2015;10(3):e011912.

21. Gathigi, Lucy Nyandia. Factors Influencing Utilization of Iron and Folic Acid Supplementation Services among Women Attending Antenatal Clinic at Nyeri Provincial Hospital Kenya. Diss. 2013.

22. Niang $\mathrm{K}$, et al. Determinants of Iron Consumption among Pregnant Women in Southern Senegal. Open J Obstet Gynaecol. 2017;7:41-50. http://www. scirp.org/journal/ojog ISSN Online: 2160-8806.

23. Alene, A, Mohamed Dohe A. Prevalence of Anemia and Associated Factors among Pregnant Women in an Urban Area of Eastern Ethiopia. Hindawi
Publ Corp Anemia Vol. 2014;2014:561567, 7 pages. https://doi.org/10.1155/2 014/561567.

24. Adediran A, et al. Haemoglobin and ferritin concentrations of pregnant women at term. Obstet Med. 2011;4(4):152-5. https://doi.org/10.1258/om.2 011.110033

25. Berhe $B$, et al. Prevalence of anemia and associated factors among pregnant women in Adigrat General Hospital, Tigrai, northern Ethiopia. BMC Res Notes. 2019;12:310. https://doi.org/10.1186/s13104-019-4347-4.

26. Gebreweld, A, Tsegaye A. Prevalence and Factors Associated with Anemia among Pregnant Women Attending Antenatal Clinic at St. Paul's Hospital Millennium Medical College, Addis Ababa, Ethiopia. Hindawi Adv Hematol Vol. 2018;2018:3942301, 8 pages. https://doi.org/1 0.1155/2018/3942301

27. Bolka A, Gebremedhin S. Prevalence of intestinal parasitic infection and its association with anemia among pregnant women in Wondo Genet district, Southern Ethiopia: a cross-sectional study. BMC Infectious Dis. 2019;19(1): 483. https://doi.org/10.1186/s12879-019-4135-8.

28. Uneke CJ, et al. Prevalence and Public-health Significance of HIV Infection and Anaemia among Pregnant Women Attending Antenatal Clinics in South-eastern Nigeria. J Health Popul Nutr 2007;25(3):328-335 ISSN 1606$0997 \mid \$ 5.00+0.20$.

29. Derso T, et al. Magnitude and associated factors of anemia among pregnant women in Dera District: a cross-sectional study in northwest Ethiopia. MC Res Notes. 2017;10:359. https://doi.org/10.1186/s13104-017-2690.

30. Wanjiru Nduhiu-Githinji. Prevalence of anaemia among pregnant women attending antenatal clinic at Mbagathi District Hospital, Nairobi. international Conference on Womens Health, Gynecology \& Obstetrics. July 08-10, 2014 Chicago NorthShore, USA

31. Intiful FD, et al. Anaemia in pregnant adolescent girls with malaria and practicing pica. Pan African Medical J. 2016;24:96. https://doi.org/10.11604/ pamj.2016.24.96.9282.

32. Getachew $M$, et al. Anaemia and associated risk factors among pregnant women in Gilgel Gibe dam area, Southwest Ethiopia. Parasit Vectors. 2012;5: 296 http://www.parasitesandvectors.com/content/5/1/296. https://doi.org/1 0.1186/1756-3305-5-296

33. Grum T, et al. Magnitude and factors associated with anemia among pregnant women attending antenatal care in public health centers in central zone of Tigray region, northern Ethiopia: a cross sectional study. BMC Pregnancy Childbirth. 2018;18:433. https://doi.org/10.1186/s12884-01 8-2063-z.

34. Judith K, Anchang-Kimbi, et al. Coinfection with Schistosoma haematobium and Plasmodium falciparum and Anaemia Severity among Pregnant Women in Munyenge, Mount Cameroon Area: A Cross-Sectional Study. Hindawi Publ Corp J Parasitol Res Vol. 2017:6173465, 12 pages. https://doi.org/10.11 55/2017/6173465

35. Stephens JK, et al. Prevalence of peripheral blood parasitaemia, anaemia and low birth weight among pregnant women in a suburban area in coastal Ghana. https://doi.org/10.11694/pamj.supp.2014.17.1.3541.

36. Kefiyalew F, et al. Anemia among pregnant women in Southeast Ethiopia: prevalence, severity and associated risk factors. BMC Res Notes. 2014;7:771 http://www.biomedcentral.com/1756-0500/7/771.

37. Lealem $\mathrm{G}$, et al. Anemia and associated factors among pregnant women attending antenatal care clinic in Wolayita Sodo town. South Ethiop. 2015. https://doi.org/10.4314/ejhs.v25i2.8.

38. Lebso $\mathrm{M}$, et al. Prevalence of anemia and associated factors among pregnant women in Southern Ethiopia: A community based cross-sectional study. PLOS ONE. 2017;12:e0188783.

39. Mengist HM, et al. Intestinal helminthic infection and anemia among pregnant women attending ante-natal care (ANC) in East Wollega, Oromia, Ethiopia. BMC Res Notes. 2017;10:440. https://doi.org/10.1186/s13104-017-2 770-y.

40. Nega $D$, et al. Anemia associated with asymptomatic malaria among pregnant women in the rural surroundings of Arba Minch Town, South Ethiopia. BMC Res Notes. 2015;8:110. https://doi.org/10.1186/s13104-015-1 081-4.

41. Obse N, Mossie A, Gobena T. Magnitude of anemia and associated risk factors among pregnant women attending antenatal care in Shalla Woreda, West Arsi Zone, Oromia Region, Ethiopia. Ethiopian journal of health sciences. 2013;23(2):165-73.

42. Nonterah EA, Adomolga E, Yidana A, et al. Descriptive epidemiology of anaemia among pregnant women initiating antenatal care in rural Northern 
Ghana. Afr J Prim Health Care Fam Med. 2019;11(1). https://doi.org/10.4102/ phcfm.v11i1.1892.

43. Ononge $\mathrm{S}$, et al. Haemoglobin status and predictors of anaemia among pregnant women in Mpigi, Ugand. BMC Res Notes. 2014;7:712 http://www. biomedcentral.com/1756-0500/7/712.

44. Ouédraogo $\mathrm{S}$, et al. Malaria and gravidity interact to modify maternal haemoglobin concentrations during pregnancy. Malar J. 2012;11:348 http:// www.malariajournal.com/content/11/1/348.

45. Obai $\mathrm{G}$, et al. Prevalence of anaemia and associated risk factors among pregnant women attending antenatal care in Gulu and Hoima Regional Hospitals in Uganda: A cross sectional study. BMC Pregnancy Childbirth. 2016;16:76. https://doi.org/10.1186/s12884-016-0865-4.

46. Shitie D, et al. Anemia and other hematological profiles of pregnant women attending antenatal care in Debre Berhan Referral Hospital, North Shoa, Ethiopia. BMC Res Notes. 2018;11:704. https://doi.org/10.1186/s13104-018-3 805-8.

47. Okube OT, et al. Prevalence and Factors Associated with Anaemia among Pregnant Women Attending Antenatal Clinic in the Second and Third Trimesters at Pumwani Maternity Hospital, Kenya. Open J Obstet Gynecol. 2016;6:16-27 Published Online January 2016 in SciRes. http://www.scirp.org/ journal/ojog. https://doi.org/10.4236/ojog.2016.61003.

48. Wells GA, Shea B, O'Connell D, Peterson J, Welch V, Losos M, Tugwell P. The Newcastle-Ottawa Scale(NOS) for assessing the quality of nonrandomized studies in meta-analyses. Appl Eng Agric. 2014;18(6):727-34.

49. Alwan NA, Cade JE, MCArdle HJ, Greenwood DC, Hayes HE, Simpson NAB. Maternal iron status in early pregnancy and birth outcomes: insights from the Baby's vascular health and Iron in pregnancy study. Br J Nutr. 2015; 113(12):1985-92. https://doi.org/10.1017/S0007114515001166.

50. Dewey KG, Oaks BM. U-shaped curve for risk associated with maternal hemoglobin, iron status, or iron supplementation. Am J Clin Nutr. 2017; 106(suppl_6):1694S-702S.

51. Sumitra M, Kumari T. Determinants of anemia among Pregnant Women attending in a tertiary level Hospital. Kathmandu Nepal. 2017;2(1):24-33.

52. Scholl TO, Hedige ML. Anemia and iron-deficiency anemia: compilation of data on pregnancy outcome. Am J Clin Nutr. 1994;59(2):492S-501S.

53. Wardlaw T, et al. United Nations Children's fund and World Health Organization. Country, Regional and Global Estimates: Low Birthweight; 2005.

54. Annotti LL, et al. Iron deficiency anemia and depleted. J Nutr. 2005;135(11): 2572-7.

55. Huang, L, Purvarshi, G, Wang, S, Zhong, L, Tang, H. The Influence of Irondeficiency Anemia during the Pregnancy on Preterm Birth and Birth Weight in South China. J Food Nutr Res. 2015;3(9):570-4. https://doi.org/10.12691/ jfnr-3-9.

\section{Publisher's Note}

Springer Nature remains neutral with regard to jurisdictional claims in published maps and institutional affiliations.

Ready to submit your research? Choose BMC and benefit from:

- fast, convenient online submission

- thorough peer review by experienced researchers in your field

- rapid publication on acceptance

- support for research data, including large and complex data types

- gold Open Access which fosters wider collaboration and increased citations

- maximum visibility for your research: over $100 \mathrm{M}$ website views per year

At BMC, research is always in progress.

Learn more biomedcentral.com/submissions 